\title{
Gross hepatomegaly due to 'minimal change' liver disease in a young female alcoholic
}

\author{
SISIR K. MAJUMDAR* + \\ B.Sc., M.B., B.S.(Cal) \\ G. K. SHAW† \\ F.R.C.Psych., D.P.M. \\ ALLAN D. THOMSON*。 \\ B.Sc., M.B., Ch.B., M.R.C.P., Ph.D. \\ P. O'GORMAN* \\ M.D., M.R.C.Path. \\ E. J. APS $\ddagger$ \\ M.B., Ch.B., M.R.C.Path. \\ J. BUGLER** \\ M.B., Ch.B., D.M.R.D. \\ ${ }^{*}$ Greenwich District Hospital, London SE10, †Elmdene Alcoholic Treatment \& Research Unit, Bexley Hospital, \\ Bexley, Kent, 'King's College Hospital Medical School, London, \\ $\ddagger$ Queen Mary’s Hospital, Sidcup, Kent and ${ }^{* * B}$ Brook General Hospital, London SE18
}

\begin{abstract}
Summary
The case of a grossly enlarged liver due to alcohol excess in a woman of 21 is reported. This case further demonstrates that a chronic alcoholic can have gross hepatomegaly with normal histology and normal liver function tests. The possible pathogenetic basis of ethanol-induced hepatomegaly ('minimal change' liver disease) is discussed.

\section{Introduction}

Hepatomegaly due to a number of well recognized pathological processes commonly occurs in alcoholism. We report a case of ethanol-induced hepatomegaly with normal liver histology and biochemistry in a woman of 21 years of age.

\section{Case report}

The patient, an unmarried English woman, was admitted in November 1980 for conventional detoxification treatment for an ethanol withdrawal syndrome. She had started social drinking at the age of 14 but admitted to drinking heavily for the last one year (about $\frac{3}{4}$-bottle of vodka daily). There was no history of drug addiction and she was not working in the alcohol trade. Her parents were both social drinkers. On clinical examination she was well nourished and the only abnormality detected was gross hepatomegaly 5 fingers breadths below the costal margin without splenomegaly or any stigmata of liver disease.
\end{abstract}

\section{Investigations}

Routine haematological profile, prothrombin time, serum folate, alpha fetoprotein and autoantibodies, conventional biochemical liver function tests, plasma urea, creatinine and electrolyte concentrations were all within normal limits. An Australia antigen test was negative. The serum $B_{12}$ level was found to be slightly raised at $1250 \mathrm{ng} /$ litre (normal $160-900 \mathrm{ng} /$ litre) and red cell folate slightly reduced at 158 ng/litre (normal 160-640 ng/litre).

Straight X-ray of the abdomen showed the liver enlarged up to the iliac crest (Fig. 1).

Liver and spleen scintigram showed an enlarged liver with increased activity in the spleen relative to the liver (Fig. 2). Liver ultrasound showed no specific diagnostic lesion and the biliary system was not dilated.

Liver biopsy showed histologically normal liver (Fig. 3a and b). The architecture on reticulin staining was normal. The parenchyma was also normal and there was no evidence of fatty change, hepatitis or cirrhosis. There was no excess stainable iron in the biopsy specimen; orcein staining of the tissue was negative. Electron microscopic study of the biopsy specimen was not done.

Following discharge she remained totally abstinent for only a few weeks and started drinking heavily again. She was readmitted in September 1981 when straight X-ray of the abdomen still showed a grossly enlarged liver to the same extent as observed on her earlier admission. Conventional liver function tests, prothrombin time and routine haematological profile were still found to be within normal limits. An indocyanine green clearance study $(0.5 \mathrm{mg} / \mathrm{kg}$ body weight) performed with a Dichromatic Ear Densitometer showed a reduced plasma half life $\left(t \frac{1}{2}\right)$ of the dye of 1.8 min; (normal 3-4 min) and an above normal 


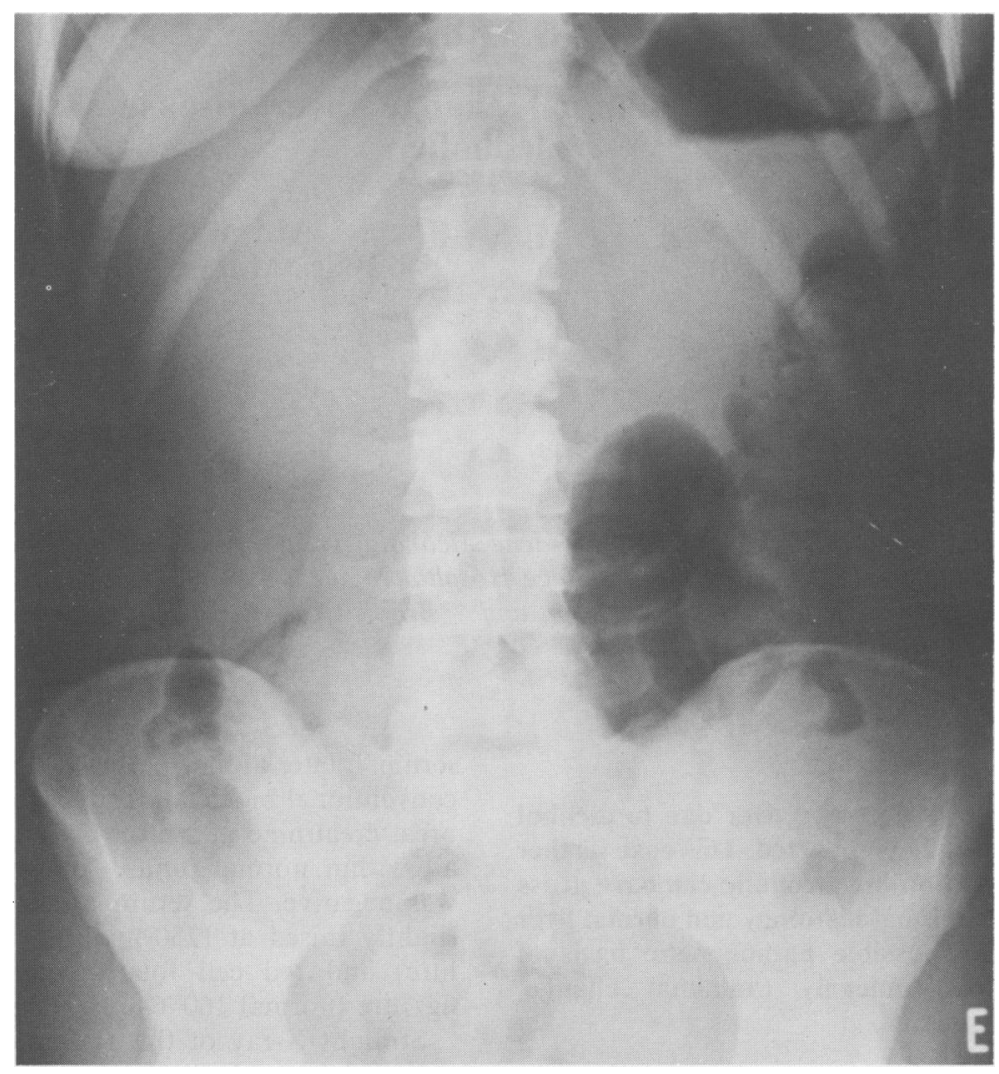

FIG. 1. Enlarged liver in straight X-ray of abdomen.

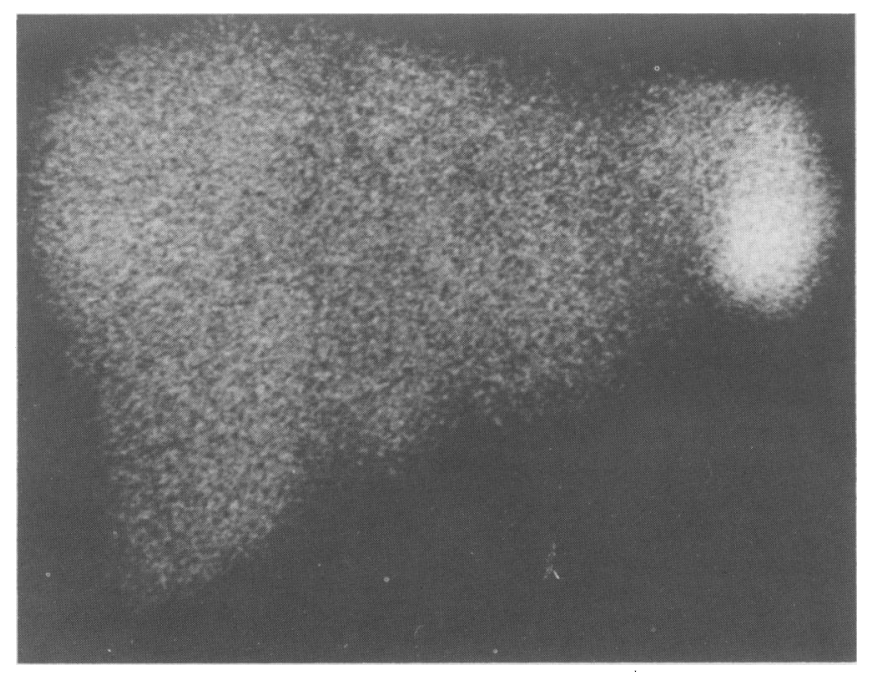

F1G. 2. Liver and spleen scintigram showing enlarged liver with normal spleen but with increased splenic activity relative to the liver. 

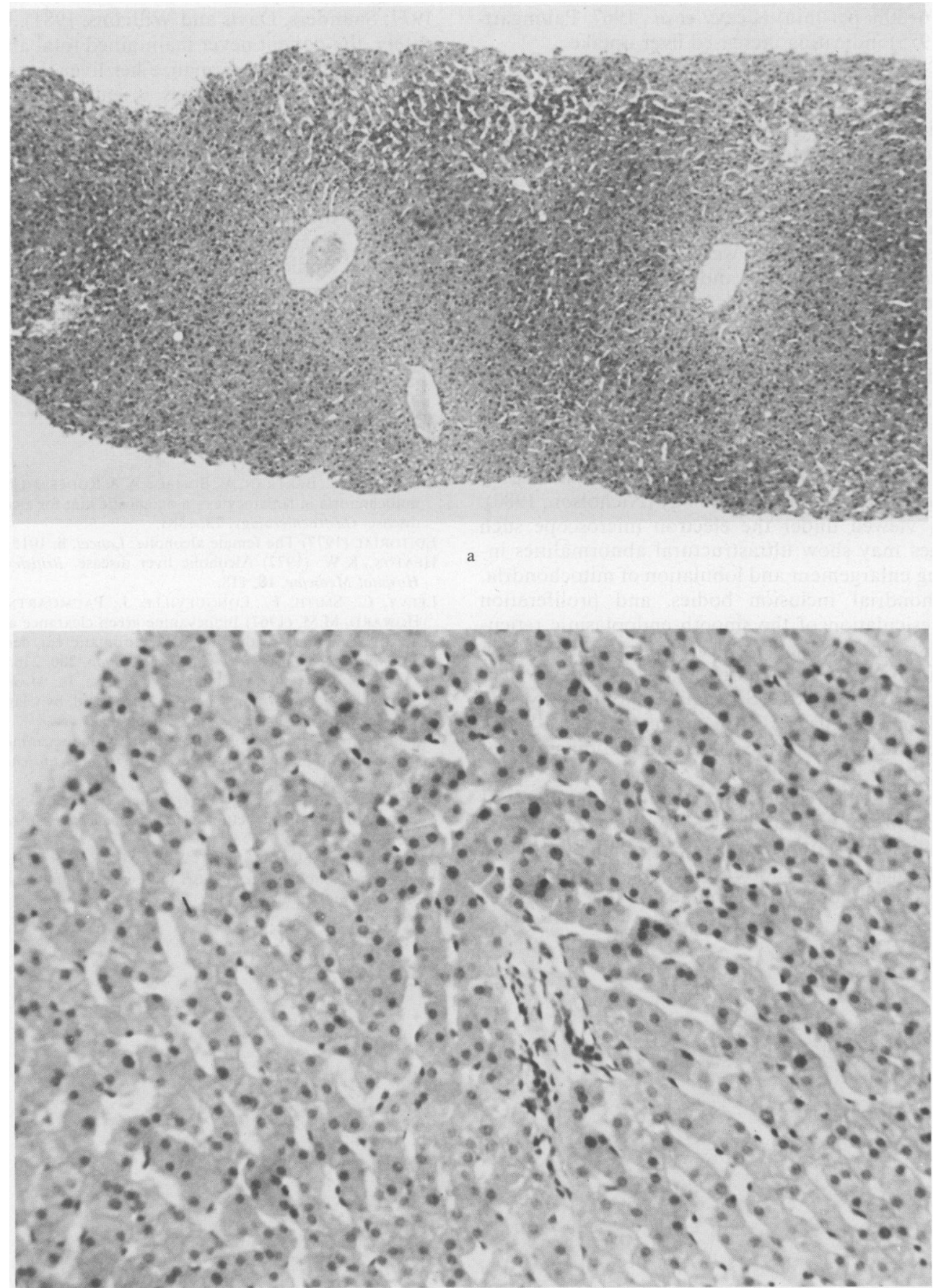

b

FIG. 3. (a) Histology of liver cells $(\mathrm{HE}, \times 50$ )

(b) Liver biopsy showing normal histology $(\mathrm{HE}, \times 125)$ 
percentage disappearance rate (PDR) of $38.5 \%$ (normal 16-30\% per min) (Leevy et al., 1967; Paumgartner, 1975) indicating increased liver uptake.

\section{Discussion}

This case is of interest because of the gross hepatomegaly at such a young age with an apparently short history of heavy drinking. Although enlarged clinically and radiologically, the liver was histologically and physiologically normal. Other causes of hepatomegaly were excluded on clinical, radiological, biochemical and histological grounds.

The patient's hepatic cells looked normal histologically on both light microscopy and on routine histochemical staining. The enlargement was probably due to adaptive hypertrophy of the intra-cellular organelles in hepatocytes which could be visible only under electron microscopy (Heaton, 1977). Such liver biopsies from alcoholic patients have been designated as showing 'minimal change' liver disease by analogy with renal histopathology (Nicholson, 1980). When viewed under the electron microscope such biopsies may show ultrastructural abnormalities including enlargement and lobulation of mitochondria, mitochondrial inclusion bodies, and proliferation and vesiculation of the smooth endoplasmic reticulum (Rubin and Lieber, 1967). Giant mitochondria (megamitochondria) can also be recognised on electron microscopy as PAS-negative, globular hyaline cytoplasmic inclusions (Bruguera et al., 1977). When all these features are present, a clinical diagnosis of alcoholism can be confirmed.

This particular case further confirms the statistical impression in epidemiological studies that female alcoholics are more susceptible to ethanol-induced liver damage with a shorter duration of drinking and lesser amount of ethanol intake for the same liver damage as compared to male alcoholics (Editorial, 1977; Saunders, Davis and Williams, 1981). Unfortunately, the patient never maintained total abstinence for long enough to normalize her liver size. Whilst a hospital stay of 7-10 days is enough to alleviate withdrawal symptoms, it is not long enough to bring back an enlarged liver to its normal size. She remained totally abstinent for only a few weeks but started drinking heavily again. Radiologically and clinically the liver was still as grossly enlarged as before. Conventional liver function tests and hepatic clearance studies showed normal excretory function of parenchymal cells although it is possible that the enlarged size of the liver compensated in some way for impaired cellular function per unit weight of the hepatocytic mass.

\section{References}

Bruguera, A., Bertran, A., Bombi, J.A. \& Rodes, J. (1977) Giant mitochondria in hepatocytes-a diagnostic hint for alcoholic liver disease. Gastroenterology, 73, 1383.

EDITORIAL (1977) The female alcoholic. Lancet, ii, 1015.

HEATON, K.W. (1977) Alcoholic liver disease. British Journal of Hospital Medicine, 18, 118.

Leevy, C., Smith, F., Longueville, J., Paumgartner, G. \& HOWARD, M.M. (1967) Indocyanine green clearance as a test for hepatic function. Evaluation by dichromatic ear densitometry. Journal of the American Medical Association, 200, 236.

Nicholson, G. (1980) Alcoholic liver disease. In: Medical Consequences of Alcohol Abuse, Ch. 4, pp. 51-86 (Ed. by Clarke, P.M.S. \& Kricka, L.J.) Ellis Horwood, Chichester.

PaUmGartner, G. (1975) The handling of indocyanine green by the liver. Supplementum. Schweizerische medizinische Wochenschrift, 105, 1.

Rubin, E. \& LIEBER, C.S. (1967) Experimental alcoholic hepatic injury in man: ultrastructural changes. Federation Proceedings, 26, 1458.

Saunders, J.B., Davis, M. \& Williams, R. (1981) Do women develop alcoholic liver disease more readily than men? British Medical Journal, 282, 1140. 